\title{
Characterization of Adaptable Interpreted-DSML
}

\author{
Eric Cariou, Olivier Le Goaer, Franck Barbier, and Samson Pierre \\ Université de Pau / LIUPPA, PauWare Research Group, BP 1155, \\ F-64013 PAU CEDEX, France \\ \{firstname.name\}@univ-pau.fr, http://www . pauware.com
}

\begin{abstract}
One of the main goals of model-driven engineering (MDE) is the manipulation of models as exclusive software artifacts. Model execution is in particular a means to substitute models for code. More precisely, as models of a dedicated domain-specific modeling language (DSML) are interpreted through an execution engine, such a DSML is called interpreted-DSML (i-DSML for short). On another way, MDE is a promising discipline for building adaptable systems based on models at runtime. When the model is directly executed, the system becomes the model: This is the model that is adapted. In this paper, we propose a characterization of adaptable i-DSML where a single model is executed and directly adapted at runtime. If model execution only modifies the dynamical elements of the model, we show that the adaptation can modify each part of the model and that the execution and adaptation semantics can be changed at runtime.
\end{abstract}

Keywords: model execution, adaptation, i-DSML, models at runtime

\section{Problem of Interest}

As programming languages, domain-specific modeling languages (DSML) can be compiled or interpreted. This distinction was early noticed by Mernik et al. [14] when comes the time to choose the most suitable implementation approach for executable DSML:

- Compiled DSML: DSML constructs are translated to base language constructs and library calls. People are mostly talking about code generation when pointing at this approach;

- Interpreted DSML: DSML constructs are recognized and interpreted using an operational semantics processed by an execution engine. With this approach, no transformation takes place, the model is directly executable.

With interpreted domain-specific modeling languages (the term i-DSML is coined in [7]), the ability to run a model prior to its implementation is a timesaving and henceforth cost-saving approach for at least two reasons: (a) It becomes possible to detect and fix problems in the early stages of the software development cycle and (b) ultimately the implementation stage may be skipped. 
One slogan associated to i-DSML should be "what you model is what you get" (WYMIWYG). Meanwhile, software adaptation and self-adaptive software [16] have gained more and more interest. Consequently, when building such software based on i-DSML, the model has to be adaptable at runtime thus requiring to define adaptable i-DSML.

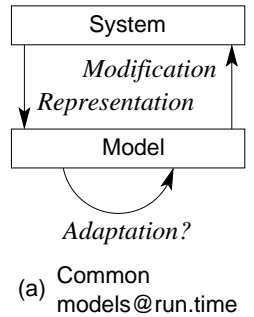

a) models@run.time

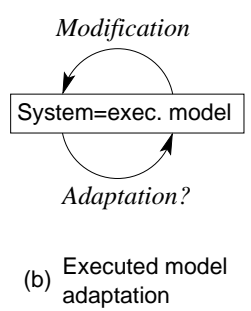

adaptation

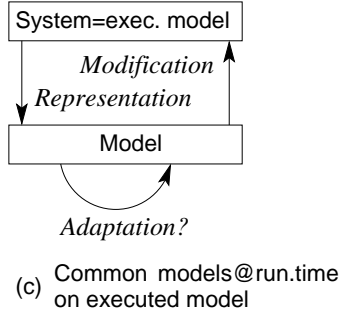

(c) on executed model

Fig. 1. Adaptation loops

The runtime adaptation problem is commonly tackled as a 2-stages adaptation loop (analyze-modification). In the MDE (Model-Driven Engineering) field, one of the most prominent way to implement this loop is models@run.time [2], where models are embedded within the system during its execution and acting primarily as a reasoning support (case (a) in figure 1). The main disadvantage of models@run.time deals with maintaining a consistent and causal connection between the system and the model for the model being a valid representation of the system at runtime. The i-DSML approach naturally circumvents this disadvantage since it suppresses the gap between the system and the model: The system is the model being executed. The reasoning is still made on the model but the required modifications are then directly enacted on the model without needing a representation of the system. Case (b) in figure 1 represents the adaptation loop in this new context. However, one major requirement for adaptable i-DSML is that the executed model contains all information necessary for both its execution and its adaptation. This can sometimes lead to an increasing complexity of the model and to a difficulty for managing the adaptation when mixing together adaptation and execution. In this case, one can abstract all the required information into a dedicated model for applying common models@run.time techniques on a model execution system. This solution is depicted by figure 1, case (c). The only difference with the case (a) is simply that the system is of a special kind: A model execution system. The problem of this solution is that it reintroduces the causal link that we precisely try to avoid here. So, both approaches (case (b) and (c)) are complementary and have pros and cons. Depending on the context, one approach will be more suited than the other one.

In this paper, we focus on the direct adaptation of an executed model (case (b) of figure 1). [5,6] are first investigations on this direct adaptation of executed models, that is, on adaptable i-DSML. They establish what model execution 
adaptation is and how to express, through a contract-based approach, that a model is consistent with an execution environment (if not, the model has to be adapted). Based on the same example of basic state machines and a train example, the contribution of this paper consists in proposing a conceptual characterization of adaptable i-DSML. The next section recalls what is model execution and its well-known conceptual characterization. This characterization is then extended in section 3 for describing what an adaptable i-DSML contains and is based on. If model execution only modifies the dynamical elements of the model, we show that the adaptation can modify each part of the model and that the execution and adaptation semantics can be changed at runtime. Finally, related word is discussed before concluding.

\section{Characterization of i-DSML}

Defining executable models is not really a novel idea. Several papers have already studied model execution, such as $[3,4,7,8,9,10,13,15]$. All these works establish a consensus about what the i-DSML approach assumes:

- Executing the model makes sense. This is much more empirical evidence that shows us that some kinds of model are executable, others are not;

- An engine is responsible for the execution of the model, that is, its evolution over time;

- The model comes with all the information necessary for its execution through an engine: It is self-contained.

Before characterizing precisely what an i-DSML contains, we give a better understanding of these three assumptions.

\subsection{Executable Nature of Models}

It exists a general classification of models that may help us to identify models which have the ability to be executed or not: The product-process duality. Indeed, models (and meta-models thereof) can either express products or processes, regardless of the system studied. By essence, only process models enable executability of their content since they embody concepts closely related to the world of runtime: Startpoint, endpoint, time (past/current/future), evolution step, etc.

Applied to the field of software development standards, we can cite SPEM as a process modeling language and CMW as a product modeling language. As another OMG's prominent example, UML itself provides three categories of diagrams, namely structure diagrams (Class, Package,...), behavior diagrams (State Machines, Activity, ...) and interaction diagrams (Communication, Sequence, ... ). Logically, only behavior and interaction diagrams may be executed. Beyond these specific examples, when designing a DSML, it is important to keep in mind its potential executable nature. 


\section{$2.2 \quad$ Execution Engines}

An i-DSML is more than just a meta-model (abstract syntax and well-formedness rules). A language definition also contains a concrete syntax and semantics. The semantics of the language are captured in the transformation rules in the case of compiled DSML or in the execution engines in the case of interpreted DSML. An execution engine is dedicated to a single i-DSML (UML state machines, SPEM, Petri nets, etc.) and can execute any model conforming to this i-DSML.

The purpose of any execution engine is to "play" or to "animate" the model, making its state evolving, step by step. Execution operations, implemented by the execution engine and potentially attached to an element of the meta-model, manage each execution step of the model. The current state of the model can be maintained locally within the engine or, differently, embedded into the model itself. The i-DSML approach singles out having self-contained models embedding their current state but the former solution can be useful in some cases. Typically, this is when one requires to execute models conforming to a meta-model not designed for managing the current state of the model, such as all the dynamic diagrams of UML. Indeed, the UML standard does not define a current model state for any of these diagrams that could be executable. In this case, the solution is to store the current state of the model within the memory of the engine or to extend the meta-model for managing a current model state. For instance, [4] did it for UML state machines. However, the extended meta-model differs from the UML standard.

\subsection{Self-contained Executable Models}

When self-contained, the current state of the model being executed is stored in the model itself. Thus, each execution step changes this state. At first glance, this strategy seems to pollute the meta-model with many details not relevant at design-time and seems to defeat the abstraction offered by traditional modeling principles (a model is the abstraction and a simplification of a given system). However, there are two main reasons justifying to have self-contained models.

The first one is that it offers the major advantage that after each execution step the current model state can be serialized into an output file ${ }^{1}$, thereby providing a complete traceability of the execution as a sequence of models. Some works, such as [3], even consider that the model can embed its complete execution trace in addition to its current state. Based on this sequence of snapshots, one can perform some useful operations like rollbacks, runtime verification (such as the black-box execution verification of [4]), debugging, testing, and so forth.

The second and main reason is related to the essence of the executable models. Such models aim at being substituted to the code, at the lowest level, far away from abstract design models. Hence they have an increased level of details and complexity required for their executability. Moreover, executability being

\footnotetext{
${ }^{1}$ That is why some authors may consider an execution process just as a sequence of endogenous model transformations, as explained in [4].
} 
part of their nature, it is unsurprising that they contain elements related to executability such as the definition of their current state.

\subsection{The Design of an i-DSML}

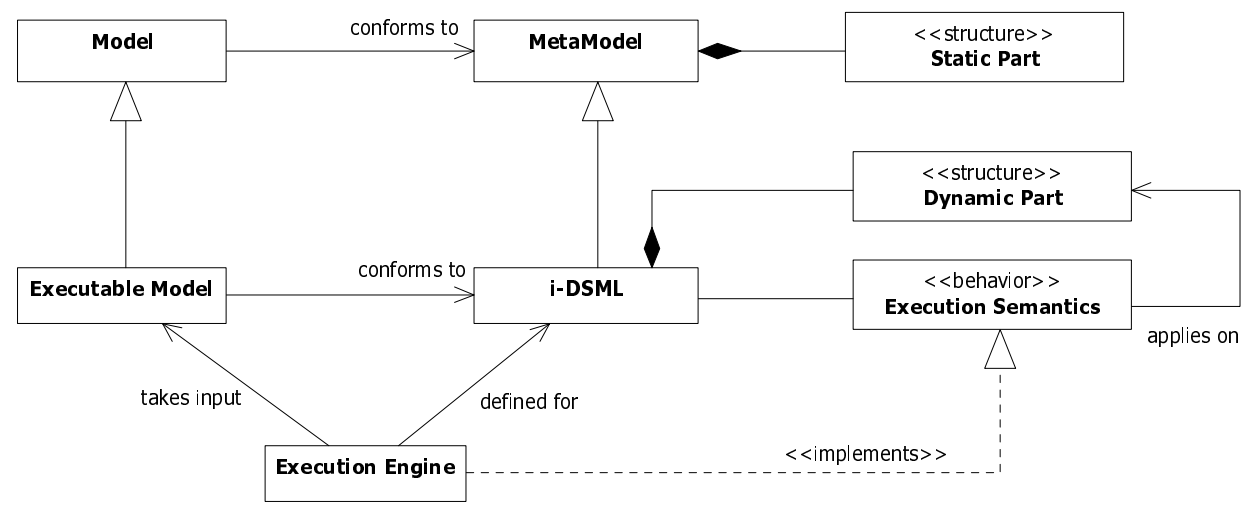

Fig. 2. Conceptual framework for an i-DSML

All elements required for model execution are located at the meta-level. Indeed, a language is a self-contained i-DSML if one can instantiate models that are executable through an execution engine. One can identify a recurring pattern [9] about the constituents of an i-DSML (figure 2):

1. Meta-elements which express the organization of the process modeled,

2. Meta-elements which express the state of the model being executed,

3. Execution semantics which express how the model behaves when being executed.

The item (1) is realized by traditional meta-modeling since the engineers concentrate onto the static structure and associated well-formedness rules of the models to be built. This is called the Static Part. Item (2) introduces structural meta-elements intended to store the state of the model at a given point of the time, also associated with their well-formedness rules. This is called the Dynamic Part. Last but not least, item (3) deals with defining how the the model is evolving over time, modifying only the dynamic part (i.e. the static part never changes). An execution semantics can be defined under several declinations. An axiomatic semantics enables to complete the specification of the meta-model with well-evolution rules defining the constraints on how the model evolves [4]. A translational semantics can be used to apply simulation or verification techniques of another technological space, such as in [8]. Finally, an operational semantics is the operationalization of the execution behavior in terms of actions through an 
action language and is implemented by an execution engine. As depicted by the figure 2, the Dynamic Part and the Execution Semantics are specific to i-DSML.

\subsection{Executable State Machines}

UML state machines are typically one of the best examples of well-known executable models. In this paper, we then link our examples to them but, for fluency, we define concise state machines restricted to a limited number of features: Composite states with deep history states and transitions associated with an event. Moreover, UML state machines as defined by the OMG do not include a dynamic part as required for a full-model execution (however [4] proposes an extension of the UML meta-model for defining a dynamic part for state machines).

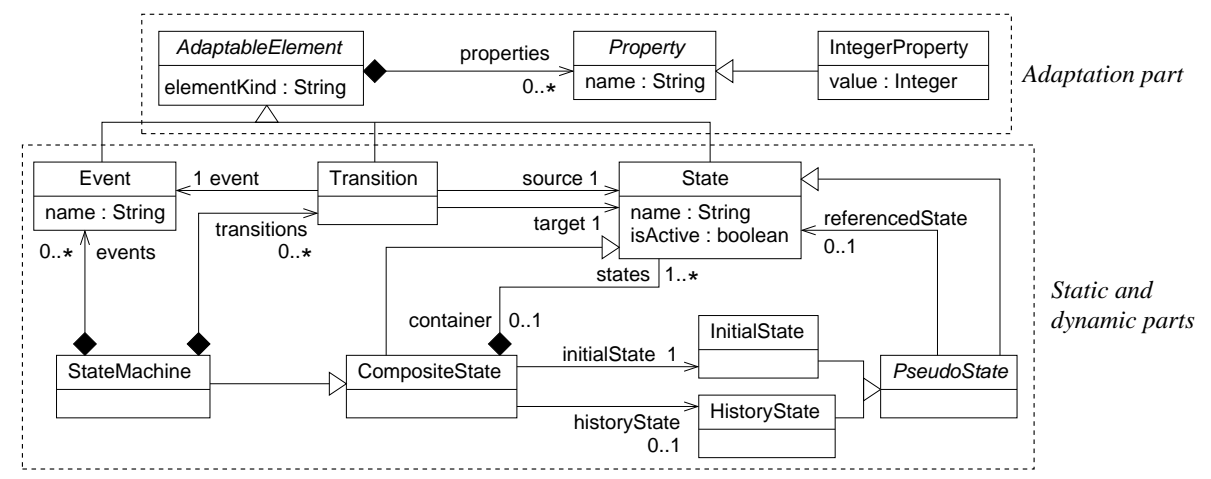

Fig. 3. Meta-model of adaptable and executable basic state machines

The meta-model of our basic state machines is represented on figure 3 (the AdaptableElement and [Integer] Property elements are dedicated for managing the adaptation and will be introduced in the next section). The static part of the meta-model is composed of the following elements:

- A state that is named and is contained in a composite state.

- Two kinds of pseudo states can be defined: An initial state and an history state, each one referencing a state of the composite state to which they belong. Each composite state must have one and only one initial state. Having an history state (but only one) is optional.

- A transition between a source and a target states, associated with an event that is simply defined with a name.

- A state machine is a special kind of composite state. It owns all transitions and events of the model and must be unique within the model.

The dynamic part of the meta-model is simply composed of two elements. The first one is the isActive boolean attribute of the State element. It enables 
to set that a state is currently active or not. The second is the referenced state of an history state that references the last active state of the composite state to which it belongs. One can note that the referencedState relation of a pseudo state is either playing a static role (for an initial state) or a dynamic one (for an history state). This state machine meta-model has been implemented in Ecore for the EMF platform.

Static and dynamic parts are complemented with OCL invariants defining the well-formedness rules for fully specifying the meta-model. For the static part, it is for instance required to express that a pseudo state references one of the states of its composite state. For the dynamic part, the main invariant specifies the active state hierarchy consistency: Either all the states are inactive (the model is not being executed) or there is in the state machine one and only one active state, and if this state is composite, it contains one and only one active state and so on.

Concerning the execution semantics, both well-evolution rules, defined using OCL, and an operational semantics, implemented by a Kermeta ${ }^{2}$ engine, have been defined. For the sake of brevity, we will not present them. Just note that their main goal is to define and to implement the main execution step ensuring that, for an event occurrence, the right transition is triggered depending on the current active states (that is, the active state hierarchy is accordingly modified).

\subsection{A Train Example}

The example of this paper is freely inspired of a railway system ${ }^{3}$. The behavior of a train is specified through a state machine. The train is stopped or is running at a given speed, depending on the light signals along the railway. The environment of execution of the train is the signals that control its speed. Concretely, the different speeds of the train are specified through the states of the state machine whereas the signals are the events associated with the transitions between these states. Within the same state machine, one can specify the behavior of the system (the train) and its interaction with the execution environment (the light signals).

Execution Environment. The train is running on railways having signals with 3 or 4 different color lights. There are two different kinds of railways: Normal speed sections (up to $130 \mathrm{~km} / \mathrm{h}$ ) and high speed sections (up to $300 \mathrm{~km} / \mathrm{h}$ ). The signal with 3 colors is for normal speed sections while the signal with 4 colors is for high speed ones. The meanings of the colors are the following (only one light is put on at the same time): red means that the train must stop immediately, amber expresses that the train must not run at more that $40 \mathrm{~km} / \mathrm{h}$, green means that the train can run at a normal speed (but not more) and purple that the train can run at a high speed.

\footnotetext{
2 http://www.kermeta.org/

${ }^{3}$ The state machines of train behaviors and their associated signals of this paper are not at all intended to be considered as realistic specification of a railway system.
} 


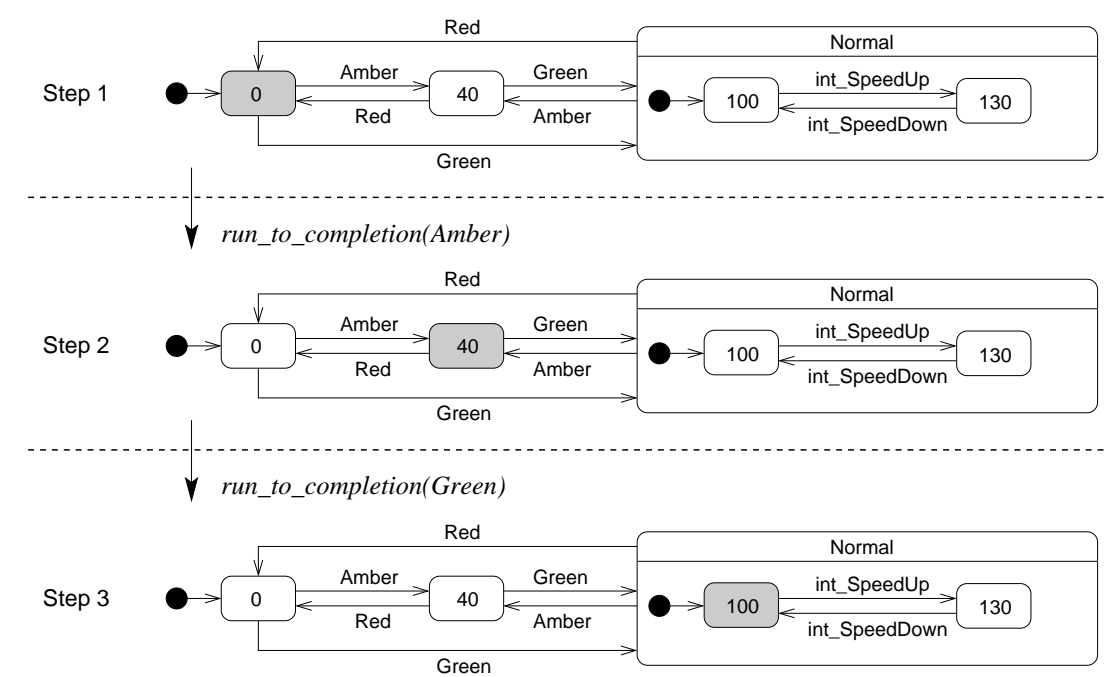

Fig. 4. A train state machine execution

Basic Train Model. The figure 4 represents a state machine defining the behavior of a non high-speed train and some steps of its execution. Concretely, this train is not able to run at more than $130 \mathrm{~km} / \mathrm{h}$ but is allowed to run at this speed on high-speed sections. The states define the speeds of the train. For simplifying, the name of a state is the train speed in $\mathrm{km} / \mathrm{h}$ associated with this state. 0 and 40 are then speeds of $0 \mathrm{~km} / \mathrm{h}$ and $40 \mathrm{~km} / \mathrm{h}$, that is the stop state and the low speed state. When running at a normal speed, the train driver can choose between two speeds, either 100 or $130 \mathrm{~km} / \mathrm{h}$. These two states have been put into a composite one representing the normal speeds. Transitions between states are associated with the signal color lights: red, green and amber. The purple color is not managed here, as the train cannot run at more than $130 \mathrm{~km} / \mathrm{h}$, but it can run at 100 or $130 \mathrm{~km} / \mathrm{h}$ on a high speed section. There are two particular events: int_SpeedUp and int_SpeedDown. These events are internal actions of the train, that is, correspond to direct train driver actions. For not confusing them with the external events coming from the execution environment, their names is prefixed by "int_."

Execution of the Train State Machine. The figure 4 shows three steps of execution of the state machine, through the run_to_completion operation taking an event name as parameter. This operation processes an event occurrence. The first step is the initial active configuration of the state machine: Its initial state 0 is active (an active state is graphically represented with a grey background). Then, for the second step, the Amber event occurs and it makes changing the current active state that is now the 40 one. Finally, the third step is the result 
of the Green event occurrence which leads to activate the Normal state and in consequence its initial state, the state 100.

Processing a state machine execution consists only in modifying the isActive attribute value for the states and, for composite states, in changing the referenced state of its potential history state. As a conclusion, only the dynamical elements of the model are modified during the execution.

\section{Characterization of Adaptable i-DSML}

We consider that an adaptable i-DSML is the logical extension of an i-DSML. Indeed, adaptable models are executable models endowed with adaptation capabilities.

\subsection{The Design of an Adaptable i-DSML}

Figure 5 depicts the design of an adaptable i-DSML. As an adaptable i-DSML is an extension of an i-DSML, this figure extends the figure 2. The added elements are:

1. Meta-elements which express properties on the model and that should help its adaptation;

2. Adaptation semantics, leveraging from the aforesaid properties, which express the adaptation problem and its solution.

Item (1) makes reference to any structural elements and their well-formedness rules that are added in the meta-model and whose role is to facilitate the subsequent adaptations. This is called the Adaptation Part. Item (2) denotes the adaptation semantics that is a specialization of an execution semantics. Indeed, while execution semantics prescribes a nominal behavior, the adaptation semantics expresses also a behavior but for extra-ordinary or abnormal situations requiring an adaptation. Again, an adaptation semantics can be declined under the specification form for complementing the meta-model definition [5], or under the operational form. As a consequence, an adaptation engine implementing the adaptation semantics is an extension of an execution engine: It processes both the execution-related operational semantics and the adaptation-related operational semantics.

Without going into details of how an adaptation semantics is managed or processed by the engine, we can say that it will mainly be composed of a set of fine-grained adaptation operations combined by a set of rules. Some operations are dedicated to checking the consistency of the model and others are actions concretely realizing the adaptation. The rules are expressed under the form "if <check> then <action>" and any more complex or recursive combination of the same kind.

The major point is that the adaptation semantics applies on elements of all constituents of the adaptable i-DSML: All the structural parts (static, dynamic 
and adaptation) and the behavioral ones (execution semantics and adaptation semantics) are concerned. Concretely, at runtime, the model's entire content can be changed including the executed semantics. This brings reflexivity to the adaptable i-DSML since enabling the adaptation of the adaptation (i.e. metaadaptation).

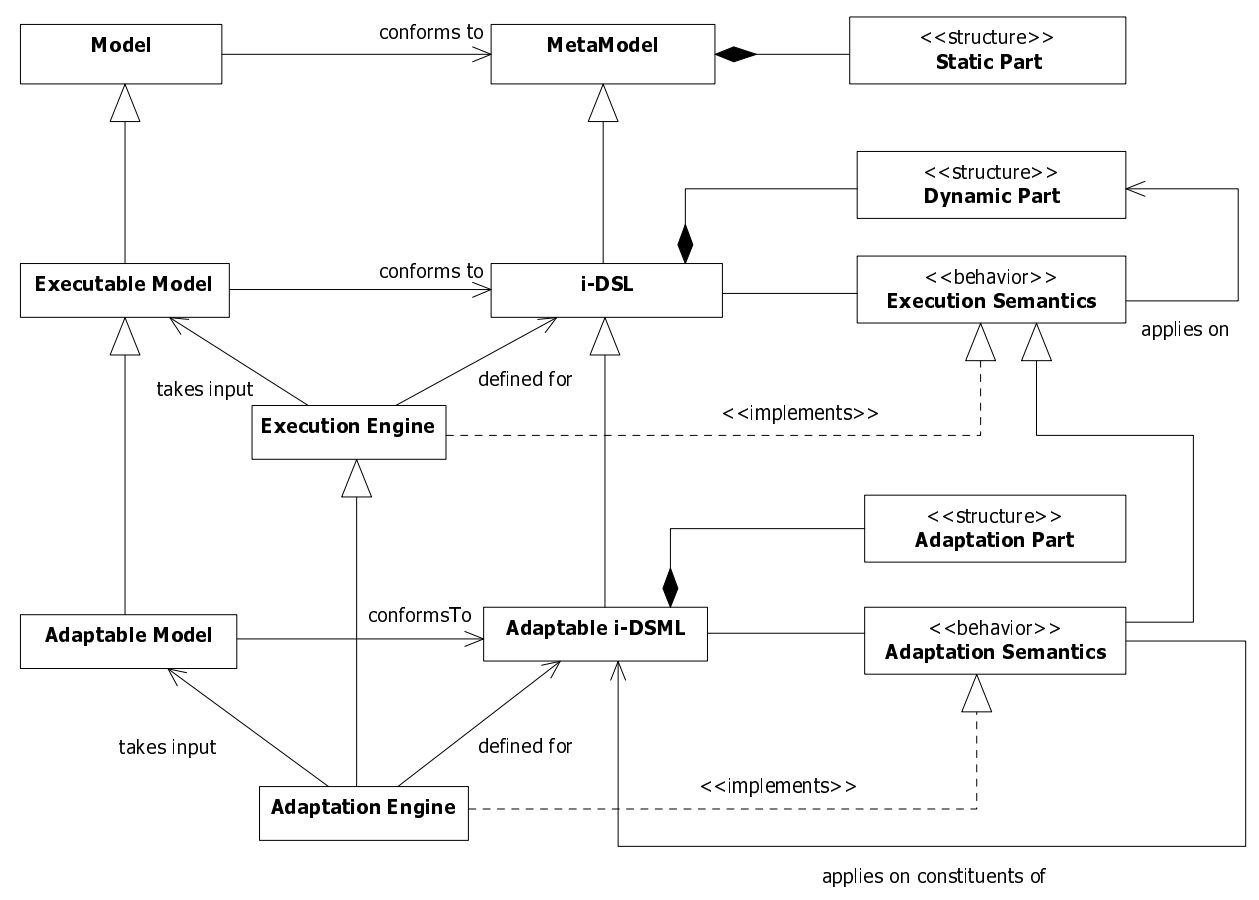

Fig. 5. Conceptual framework for adaptable i-DSML

Categories of Adaptation Actions. We identified two categories of adaptation actions: Create/Update/Delete (CUD) and Substitution. CUD actions target instances of any structural parts (static, dynamic, and adaptation). Substitution is an action which targets the behavioral parts (execution and adaptation semantics). We consider only substitution for behavioral parts because it is not feasible to define a new semantics from scratch (concretely, it will consist in writing lines of code in the engine). Instead, having a set of existing semantics (i.e. already implemented) and choosing at runtime which of them to process is straightforward. CUD and substitution can be applied with the following purposes: 
- CUD on the dynamic part: The current state of execution is altered (e.g. force to go back/go forward, restart such as activating a given state for a state machine),

- CUD on the static part: The structure of the model itself is changed (e.g. modification of the modelized process such as adding a state or changing the initial state of a composite for a state machine),

- CUD on the adaptation part: An adaptation-related element is changed (e.g. the value of a QoS property is modified accordingly to a changing execution environment),

- Substitution on the execution semantics: Switch from a given interpretation of the model to another one (e.g. managing execution variants such as the Harel vs UML transition conflict semantics for state machines),

- Substitution on the adaptation semantics: Switch from a given set of adaptation operations to other ones within the adaptation rules (e.g. checking the consistency of the model with the execution environment in an exact or fail-soft mode).

Table 1. Adaptation and execution characteristics

\begin{tabular}{|c|c|c|c|}
\hline \multicolumn{2}{|c|}{ Elements of adaptable i-DSML } & Execution actions & Adaptation actions \\
\hline \multirow{3}{*}{$<<$ Structure $>>$} & Static Part & N/A & Create/Update/Delete \\
& Dynamic Part & Create/Update/Delete & Create/Update/Delete \\
& Adaptation Part & N/A & Create/Update/Delete \\
\hline \multirow{2}{*}{$<<$ Behavior $>>$} & Execution Semantics & N/A & Substitution \\
& Adaptation Semantics & N/A & Substitution \\
\hline
\end{tabular}

Table 1 sums up these categories of adaptation actions and contrasts with the actions processed by a simple model execution. Indeed, in this case, only the dynamic part of the model is modified whereas for model adaptation, all parts and semantics can be changed.

\subsection{Adaptation Part for the State Machine Meta-Model}

The meta-model of state machines of the figure 3 includes elements dedicated to the adaptation management. The first one is the elementKind attribute available for the Event, Transition and State elements through the specialization of AdaptableElement. This attribute allows the definition of "kinds" for events, transitions and states of a state machine. A kind has for goal to precise that an element is playing a particular role. Conceptually, a kind is equivalent to a stereotype of UML profiles. In addition, through the properties association, events, transitions and states can be associated with properties. A property is 
basically composed of a name and a value. For simplicity, only integer properties are considered here, but of course, properties of any type could be added on the meta-model (the definition of properties can of course be based on reusing existing adaptation works, such as [11] which defines a generic meta-model for specifying properties and associated rules depending on their values). Properties can deal if required with QoS parameters and values. Conceptually, a property is equivalent to a tagged value of UML profiles.

As shown in the following, kinds and properties can be used for managing the adaptation of a state machine execution thanks to the additional information they offer. Kinds can be used to define fail-soft mode of consistency against an execution environment. Properties associated with events of the execution environment enable the modification of the executed state machine for managing unexpected events.

\subsection{Runtime Adaptation of the Train State Machine}

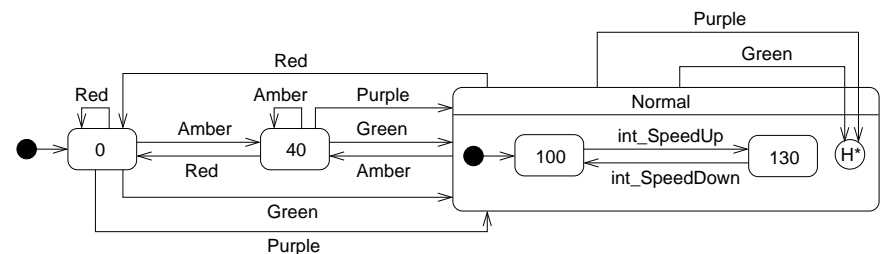

(a) Enhanced train state machine: Explicit management of all events

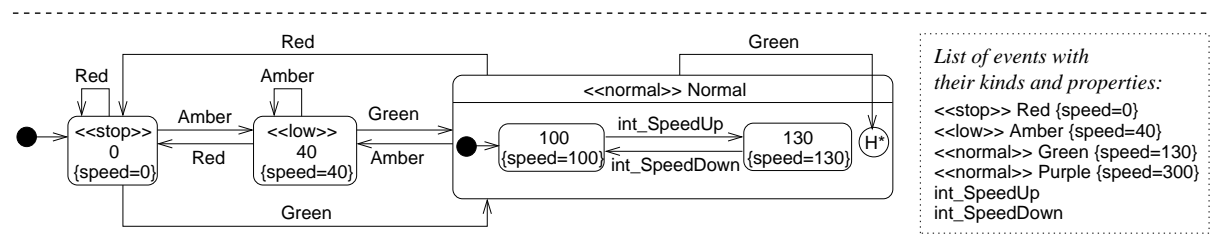

(b) Enhanced train state machine: Properties and kinds for model elements

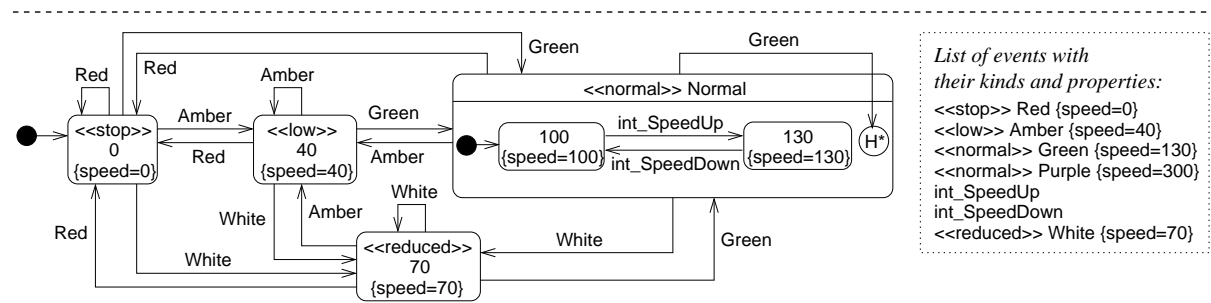

(c) Train state machine: Addition of a state and its associated transitions

Fig. 6. Adaptation of the train state machine 
The main adaptation rule consists in first checking if the behavior of the system is consistent with the execution environment, that is concretely here, if any signal is understandable and correctly processed by the train state machine. Secondly, when the system is not consistent with the environment, to perform an adaptation action such as switching in a fail-soft checking mode or modifying the structure of the model.

As explained in $[5,6]$, specific design constraints can be applied for verifying the consistency of a model against an execution environment. The problem is that it is necessary to be able to distinguish an unexpected interaction - requiring to take an adaptation decision - from an expected and already managed one. For state machines, it is required to be able to determine if an event is expected or not. Several solutions are possible, such as parameterizing the execution engine with the list of expected events and to verify for each event occurrence if it is in the list. The solution applied here is self-contained in the model by adding explicitly a transition starting from each state for each expected event. Figure 6 , part (a), shows the modification of the train state machine: For every expected color events (red, amber, green and purple), there is a transition starting from each state. When a color event leads to remain in the same state, it is a selftransition (leading to the historical state of a composite for composite states). Now, excepting for internal events starting by "int_", each event, corresponding to a color of signal crossed by the train, triggers a transition. If no transition is associated with an event according to the current active states, then this event is unexpected and an adaptation action has to be performed.

Based on this new train state machine definition, as an illustration, we describe here seven adaptation operations (two adaptation checking and five adaptation actions including one modifying the adaptation semantics) and two execution semantics variants for the state machine meta-model.

Adaptation Checking. The main verification is to determine if an event is expected or not. This verification can be made in an exact mode or in a failsoft mode through the kinds of events. Let us consider the occurrence of the purple signal event. The train is not able to run at a high speed level, so, when crossing such a purple signal, the train will run at a normal speed. For this reason, the train state machine of figure 6 part (a) leads to the state Normal for each occurrence of the purple event.

The exact mode consists in verifying that there is a transition associated with the exact event (through its name) and the fail-soft mode that there is a transition for the kind of event and not necessary for the exact event. Figure 6, part (b), shows a variant of the train state machine where kinds and properties have be added onto states and events. There are three kinds of states (the kinds are represented through the $\langle<. .>>$ UML stereotype notation): The 0 state is a stop state, the 40 one is a low speed state and the composite state Normal is a normal speed state. The events, depending of their associated target state ${ }^{4}$, are

\footnotetext{
${ }^{4}$ We rely on a dedicated restriction on the state machines: All transitions associated with a given event are always leading to the same state. For instance for the train
} 
also tagged with kinds: red is a stop event, amber is a low speed event, green and purple are normal speed events. One can notice that transitions associated with the purple color have disappeared. Indeed, in a fail-soft mode, the purple event will be processed as the green one because there come from the same normal kind. The purple color is for the train considered as a green one even if they do not have the same role and meaning.

The verification mode can be changed at runtime: A checking adaptation operation can be substituted by another one (changing in that way the adaptation semantics). If the model of the figure 6 , part (b), is executed and if the train is running on normal speed sections, then the verification mode can be the exact one because the red, amber and green signals that can be crossed are directly and exactly processed for each state of the state machine. However, if the train is now running on a high-speed section, it can cross a purple signal that is not directly processed in the exact mode. An adaptation action can be to switch into a fail-soft verification mode and to recheck the validity of the purple signal. In this mode, as explained, this signal will be considered as a green one and will be processed.

From an implementation point of view, our Kermeta execution engine has been extended for managing the adaptation. Mainly, a pre-condition has been added for the run_to_completion operation that processes each event occurrence. This pre-condition performs the chosen adaptation checking and, if not respected, an adaptation action is executed.

Adaptation Actions. In addition to substituting an adaptation checking operation by another one, several adaptation actions can be taken in case of unexpected events, that is, in case of a changing execution environment. A very basic action is to stop the execution of the state machine if there is no way to decide how to handle the event. A more relevant adaptation action is to load a new state machine (a reference one as defined in [6]) that is able to manage the new execution environment if such a state machine is available.

If the unexpected event is associated with properties, they can be used to determine if this event can target an existing state of the state machine or, if not, to add the associated state and transitions on the state machine. Figure 6, part (b), defines a speed property for each event and state. Properties are represented similarly to tagged values of UML profiles (as the $\{$ speed=XXX $\}$ ones). For instance, the amber event and the state 40 are both associated with a speed property of the value 40 , that is, $40 \mathrm{~km} / \mathrm{h}$. Let us suppose that, if this train state machine is executed, a white signal, of a reduced kind and a speed property of $70 \mathrm{~km} / \mathrm{h}$, is crossed. In both exact and fail-soft verification modes, this white event is an unexpected one. As no state has a speed property with a value of 70 , a new state called 70 is created with the same kind and speed property as the white event. All required transitions, starting from or leading to this new state, have also to be added: Each existing state must be the source of a transition

state machine, independently of the source state, the amber event always leads to the state 40 . 
associated with the white event and leading to this new state and for each color event (red, amber and green) there must be a transition starting from this new state and leading to the required state. The figure 6 , part (c), shows the resulted runtime modification for managing the white signal. An important point to notice about this model modification is that it is based on the comparison of the properties without requiring to know what they are and what they are representing. The adaptation engine simply compares two sets of properties through their names and values.

A last adaptation action could be to force the activation of the state that is from a stop kind (the state 0 for the train state machine) in case of an unexpected event (this action is different from stopping the execution of the state machine as described above because here the state machine is still being executed). The idea is that the train stops if it does not understand a signal.

Execution Semantics Variants. For state machines, a transition conflict appears when a transition is starting from a composite state and that there is also a transition associated with the same event starting from an internal state of this composite. The way to choose which transition to fire when this event occurs is a semantics variation point. According to the UML semantics ${ }^{5}$, it is the most internal one that is fired while the original Harel statecharts semantics [12] leads to fire the external one starting from the composite.

When loading a state machine model, including at runtime when changing the current state machine by another one suited for a new context of execution, it is required to know with which execution semantics it was designed. We can imagine a property associated with the state machine and precising which kind of transition processing variant must be used. Then, the execution engine embeds the operational code for each semantics and the right one is processed depending of this property value. In other words, when changing the executed model as an adaptation action, the current operational semantics can be substituted by another one if needed.

\section{Related Work}

As written, several papers such as $[3,4,7,8,9,10,13,15]$ have already studied model execution. Section 2 summarizes the consensual characterization of model execution based on these works.

Concerning the adaptation of model execution, as far as we know, there are no other works except ours which have studied this subject. $[5,6]$ have been used as a base for defining the direct model execution characterization exposed in this paper. The MOCAS platform [1] defines a UML agent state machine observing an executed business UML state machine and requiring changes on its current state or structural content. The adaptation is then made following common models@run.time adaptation techniques (case (c) of figure 1). The problem

\footnotetext{
${ }^{5}$ http://www.omg.org/spec/UML/2.2/
} 
is that the adaptation and the execution operations are strongly mixed-up in the implementation platform. This leads to the absence of separation of the adaptation logic from execution. Moreover, the platform does not enable to replace at runtime the adaptation or execution semantics as we propose.

[13] offers a characterization of models at runtime and related adaptation in the same spirit of this paper. But there are two main differences with our characterization of direct adaptation of model execution: (a) It always considers that the model, even when executable, is causally connected with a running system whilst for us the executed model is by essence the system and (b) it does not go as far as us about the elements that can be modified by the adaptation: It does not consider that the execution semantics or the adaptation semantics can be changed.

\section{Conclusion}

In this paper we propose a conceptual characterization of the direct adaptation of model execution, through the concept of adaptable i-DSML. Albeit model execution and adaptation are closely related (as an adaptation semantics is a specialized execution semantics), there are two sharp demarcation lines. The first one, from a general point of view, is about the intention embodied in the semantics. Indeed, an execution semantics deals with a nominal behavior whereas an adaptation one concerns extra-ordinary or abnormal situations. The second one, from a technical point of view, is that model execution only modifies the dynamic elements of the model whereas model adaptation can modify each part of the model and the execution and adaptation semantics.

The presented execution and adaptation engine is a first prototype showing the interest of studying the adaptation of an executed model. We need to develop more realistic and complex case studies and to consider the adaptation of other kinds of adaptable i-DSML. Notably, we plan to extend our existing tools dedicated to execute full standard UML state machines: $\operatorname{SimUML}^{6}$ is a simulation tool at the design level and PauWare ${ }^{7}$ a Java library implementing and executing UML state machines for any Java platform, including Android devices. The goal is to define and implement adaptation operations and semantics for UML state machines. We plan also to enhance our MOCAS platform for making it clearly separating the adaptation from the execution. These platforms and complex case studies will allow us to study the limits of directly adapting a model execution versus applying common models@run.time techniques on it. One of our perspective is to determine when one approach is more suited than the other one. Finally, just as an action language is provided for expressing the execution semantics, a mid-term perspective is to provide a full-fledged adaptation language. This DSML will support the adaptation loop by offering language constructs for both checking and actions. Concretely, it will enable to define separately the execution logic and the adaptation one, and then to express how the

\footnotetext{
${ }^{6}$ http://sourceforge.net/projects/simuml/

${ }^{7}$ http://www. pauware.com/
} 
adaptation checking and actions are orchestrated and weaved with the execution operations.

\section{References}

1. C. Ballagny, N. Hameurlain, and F. Barbier. MOCAS: A State-Based Component Model for Self-Adaptation. In Third IEEE International Conference on SelfAdaptive and Self-Organizing Systems (SASO '09). IEEE Computer Society, 2009.

2. G. S. Blair, N. Bencomo, and R. B. France. Models@run.time. IEEE Computer, 42(10):22-27, 2009.

3. E. Breton and J. Bézivin. Towards an understanding of model executability. In Proceedings of the international conference on Formal Ontology in Information Systems (FOIS '01). ACM, 2001.

4. E. Cariou, C. Ballagny, A. Feugas, and F. Barbier. Contracts for Model Execution Verification. In Seventh European Conference on Modelling Foundations and Applications (ECMFA '11), volume 6698 of LNCS. Springer, 2011.

5. E. Cariou and M. Graiet. Contrats pour la vérification d'adaptation d'exécution de modèles. In 1ère Conférence en IngénieriE du Logiciel (CIEL 2012), 2012.

6. E. Cariou, O. Le Goaer, and F. Barbier. Model Execution Adaptation? In 7th International Workshop on Models@run.time (MRT 2012) at MoDELS 2012. ACM Digital Library, 2012.

7. P. J. Clarke, Y. Wu, A. A. Allen, F. Hernandez, M. Allison, and R. France. Formal and Practical Aspects of Domain-Specific Languages: Recent Developments, chapter 9: Towards Dynamic Semantics for Synthesizing Interpreted DSMLs. IGI Global, 2013.

8. B. Combemale, X. Crégut, P.-L. Garoche, and T. Xavier. Essay on Semantics Definition in MDE - An Instrumented Approach for Model Verification. Journal of Software, 4(9), 2009.

9. B. Combemale, X. Crégut, and M. Pantel. A Design Pattern to Build Executable DSMLs and associated V\&V tools. In The 19th Asia-Pacific Software Engineering Conference (APSEC 2012). IEEE, 2012.

10. G. Engels, J. H. Hausmann, R. Heckel, and S. Sauer. Meta-Modeling: A Graphical Approach to the Operational Semantics of Behavioral Diagrams in UML. In the 3rd international conference on the Unified Modeling Language (UML 2000), volume 1939 of $L N C S$. Springer, 2000.

11. F. Fleurey and A. Solberg. A Domain Specific Modeling Language Supporting Specification, Simulation and Execution of Dynamic Adaptive Systems. In MODELS '09, volume 5795 of $L N C S$. Springer, 2009.

12. D. Harel. Statecharts: A visual formalism for complex systems. Science of Computer Programming, 8(3), 1987.

13. G. Lehmann, M. Blumendorf, F. Trollmann, and S. Albayrak. Meta-Modeling Runtime Models. In Models@run.time Workshop at MoDELS 2010, volume 6627 of $L N C S$. Springer, 2010.

14. M. Mernik, J. Heering, and A. M. Sloane. When and how to develop domainspecific languages. ACM Comput. Surv., 37(4), 2005.

15. C. Pons and G. Baum. Formal Foundations of Object-Oriented Modeling Notations. In 3rd International Conference on Formal Engineering Methods (ICFEM 2000). IEEE, 2000.

16. M. Salehie and L. Tahvildari. Self-adaptive software: Landscape and research challenges. ACM Trans. Auton. Adapt. Syst., 4:14:1-14:42, 2009. 